\title{
A pesquisa em educação ambiental e questões metodológicas: relato do grupo de discussão de pesquisa no vi epea
}

José Artur Barroso Fernandes ${ }^{1}$

Clarice Sumi Kawasaki ${ }^{2}$

Resumo: No presente artigo, sistematizamos as reflexões feitas pelos participantes de um Grupo de Discussão de Pesquisa (GDP) recéminaugurado no VI EPEA, sobre "Questões Metodológicas da Pesquisa em Educação Ambiental”, a fim de contribuir para um mapeamento inicial dessa temática nesse evento e para uma avaliação sobre a pertinência ou não de um aprofundamento maior dessas questões em GDPs futuros. Considerações sobre o objetivo central desse GDP, as motivações que nos levaram a iniciar uma discussão sobre esta temática em GDP, o que entendemos por "questões metodológicas", como foram encaminhadas as discussões no grupo, as reflexões do grupo e as sugestões de encaminhamento dadas para a continuidade das discussões compõem os principais itens desenvolvidos neste texto.

Palavras-chave: Pesquisas em educação ambiental, Questões metodológicas, Trabalhos do VI EPEA.

\section{Research on Environmental Education and Methodological Issues: report by the Research Discussion Group at the VI EPEA}

Abstract: In this paper we systematize the reflections of the participants of a Research Discussion Group (RDG) called "Methodological Issues of the Research on Environmental Education". The group was recently included in the VI EPEA in order to contribute to providing both a preliminary mapping of that theme in the event and an assessment whether it is relevant or not to delve into such issues in future RDGs. Considerations on the main objective of that RDG, motivations that have led us to start a discussion on that theme in a RDG, what we understand by "methodological issues", how the discussions in the group were addressed,

\footnotetext{
1 Professor do Departamento de Metodologia de Ensino - DME do Centro de Educação e Ciências Humanas da Universidade Federal de São Carlos.( UFSCar -),São Carlos, Brasil, zeartur@ufscar.br

2 Professora doutora da Faculdade de Filosofia, Ciências e Letras de Ribeirão Preto (USP),Ribeirão Preto, Brasil, sumi@ffclrp.usp.br
} 
the reflections of the group, and the points made for the continuity of the discussions are the main items addressed in this text.

Keywords: Research on environmental education. Methodological issues. Works of the VI EPEA.

\section{La investigación en educación ambiental y cuestiones metodológicas: relato del grupo de discusión de investigación en el vi epea}

Resumen: En el presente artículo, sistematizamos las reflexiones hechas por los participantes de un Grupo de Discusión de Investigación (GDP) recién inaugurado en el VI EPEA, sobre "Cuestiones Metodológicas da Pesquisa en Educación Ambiental”, a fin de contribuir para un mapeamiento inicial de esa temática en ese evento y para una evaluación sobre la pertinencia o no de una profundización mayor de esas cuestiones en GDPs futuros. Consideraciones sobre el objetivo central de ese GDP, las motivaciones que nos llevaron a iniciar una discusión sobre esta temática en GDP, lo que entendemos por "cuestiones metodológicas", cómo fueron encaminadas las discusiones en el grupo, las reflexiones del grupo y las sugerencias de encaminamiento dadas para la continuidad de las discusiones componen los principales ítems desarrollados en este texto.

Palabras clave: Investigaciones en educación ambiental, Cuestiones metodológicas, Trabajos del VI EPEA.

\section{Introdução}

Tratar de questões metodológicas da pesquisa em Educação Ambiental (EA) parece-nos a princípio um objetivo muito abrangente e genérico, havendo a necessidade de uma definição maior sobre "quais questões metodológicas o Grupo de Discussão de Pesquisa (GDP) iria se debruçar". É nesse exercício de delimitação que decidimos, num primeiro momento, olhar para os trabalhos que foram inscritos no referido GDP, pois havia uma hipótese inicial de que esses trabalhos teriam como enfoque as questões metodológicas, ou seja, as questões de pesquisa estariam voltadas para os aspectos metodológicos da pesquisa em EA. Num segundo momento, nos caberia olhar para o conjunto de 88 trabalhos aprovados no VI EPEA do ponto de vista das opções metodológicas neles contidas. Assim, delimitaríamos de certo modo o enfoque dado a esse GDP e, ao mesmo tempo, garantiríamos uma visão panorâmica dessas questões no conjunto dos trabalhos aprovados no VI EPEA.

Elegemos essa temática para ser discutida em um novo GDP surgiu em razão de demandas internas, relacionadas à necessidade de um destaque maior a 
essas questões visando o seu aprofundamento, conforme será apontado em seguida no percurso feito pelos cinco EPEAs já realizados, e demandas externas, já que no cenário educacional como um todo, e na educação ambiental em especial, as questões metodológicas vêm sendo destacadas, em virtude da multiplicidade de objetos de estudo, de áreas do conhecimento e de sujeitos envolvidos. $O$ entrelaçamento dessa multiplicidade de aspectos certamente demandaria novas metodologias, conforme veremos mais adiante nas considerações feitas por Gatti (2010) sobre a complexidade do campo da pesquisa em educação.

Analisando as temáticas dos cinco EPEAs, verificamos um aprofundamento gradativo nas questões filosóficas, epistemológicas e metodológicas do campo da pesquisa em EA, iniciando, em 2001 (Rio Claro), com um diagnóstico do estado da arte das pesquisas em EA (CARVALHO et al., 2001), passando em seguida, em 2003 (São Carlos), por uma discussão sobre "as abordagens epistemológicas e metodológicas" dessas pesquisas (FREITAS et al., 2003) e avançando, em 2005 (Ribeirão Preto), na direção da discussão sobre "as práticas de pesquisa em educação ambiental" (KAWASAKI, 2005). Como se pode ver, o enfoque nas abordagens epistemológicas e metodológicas já estava presente no terceiro encontro.

O tema "o debate modernidade e pós-modernidade" veio a responder, em 2007 (Rio Claro), a demandas muito presentes naquele momento, quando as discussões sobre o pós-modernismo como contraponto importante e crítica ao modernismo vigente estavam fervilhando, marcando posições diferenciadas e disputas no campo da pesquisa em EA (CARVALHO et al., 2007).

Toda essa discussão acumulada veio a convergir, em 2009 (São Carlos), para a necessidade de reflexões sobre "a configuração do campo de pesquisa em EA", ou seja, a pesquisa em EA precisa ser compreendida como um campo em formação, em busca de sua identidade, do delineamento de suas questões, teorias, fronteiras e zonas de intersecção com áreas próximas, especialmente com os campos educacional e ambiental, assim como de sua institucionalização (especialmente nas pós-graduações) e da formação de profissionais. É nessa tentativa de configuração de um campo de pesquisa em EA que se busca o aprofundamento do debate sobre pressupostos epistemológicos e os fundamentos metodológicos inerentes aos processos de investigação do processo educativo de maneira geral e do processo educativo relacionado com a temática ambiental de forma particular. E é a partir dessas premissas que o presente relatório evidenciou uma conexão entre os três encontros, o que lhes confere um caráter processual de construção de conhecimento e de consolidação da área (OLIVEIRA et al., 2009).

Quanto ao VI EPEA, a temática escolhida buscou aprofundar as discussões sobre "a pesquisa em EA com foco específico dos Programas de Pós-Graduação no Brasil". Considerando que a pesquisa em EA vem se constituindo nas últimas 
duas décadas dentro de programas de pós-graduação estruturados em diferentes formatos e modelos e em diferentes áreas do conhecimento, esse evento focou a discussão da inserção da pesquisa em EA na pós-graduação do Brasil, assumindo como objetivos: a) refletir sobre a inserção da pesquisa em EA na pós-graduação do Brasil, b) discutir a influência do sistema de pós-graduação nas pesquisas em EA e c) analisar experiências concretas de pesquisas em EA em diferentes modelos de programas de pós-graduação (MATOS, 2011). E foi nessa $6^{a}$ edição do EPEA que se inaugurou esse GDP sobre "Questões Metodológicas da Pesquisa em EA".

Embora os grupos de discussão já existissem desde o primeiro encontro, no terceiro, em 2005, é que foi proposto um formato diferente para a discussão das pesquisas em EA: os Grupos de Discussão de Pesquisa (GDP). A ideia desses GDPs é oferecer aos participantes um espaço para a discussão de pesquisas, articuladas a partir de determinados eixos temáticos. Os GDPs são coordenados por pesquisadores convidados pela organização do evento por possuírem produção reconhecida na temática que irão desenvolver. A dinâmica dos trabalhos durante a realização dos GDPs é a seguinte: no início dos trabalhos, o coordenador apresenta um texto gerador e, em seguida, organiza dinâmicas participativas para envolver os participantes nas discussões e reflexões acerca da temática de pesquisa. O objetivo do texto é oferecer referenciais teóricometodológicos para as pesquisas relacionados com aquele tema e ser o passo inicial para as discussões no grupo. No último dia do encontro o coordenador do grupo apresenta para a plenária as ideias básicas do texto e uma síntese das discussões do grupo, elaborada com a ajuda de relatores escolhidos pelo próprio grupo. Após o encontro, as reflexões dos participantes dos GDPs sobre as discussões propostas pelos coordenadores são sistematizadas por estes na forma de artigos, procurando enfatizar as questões que se colocam como mais significativas para a produção de conhecimentos em cada uma das áreas temáticas trabalhadas pelos grupos. Esses artigos são publicados na revista Pesquisa em Educação Ambiental, em um número especial voltado para as produções dos EPEAs.

Os eixos temáticos desenvolvidos nos GDPs são:

GDP 1: A Educação Ambiental e as Controvérsias Sociocientíficas;

GDP 2: Educação para a Sustentabilidade: Implicações para o Currículo Escolar e para a Formação de Professores;

GDP 3: Educação Ambiental no Contexto Escolar;

GDP 4: Educação Ambiental e Intervenções Sociais;

GDP 5: Epistemologia e Ética.

Na avaliação dos participantes, em 2005, 2007 e 2009, segundo os relatórios científicos do III, IV e V EPEA, os GDPs representam uma ampliação dos 
espaços de discussão, para além das conferências, mesas-redondas e apresentações orais dos trabalhos, permitindo maior envolvimento dos participantes do evento. Considerando essa avaliação positiva dos resultados dos trabalhos dos GDPs, a Comissão Organizadora do EPEA decidiu manter essa proposta na estrutura do evento, com algumas alterações nas temáticas: permaneceram as mais abrangentes - movimentos sociais, contexto escolar, questões epistemológicas e formação de educadores/professores - e foram substituídas as demais por temáticas atuais no contexto da pesquisa em EA pesquisa-ação e percepção ambiental, em 2009, e questões metodológicas da pesquisa em EA, em 2011.

\section{O QUE SÃO “QUESTÕES METODOLÓGICAS” PARA ESSE GDP?}

Em Gatti (2010), encontramos citação de H. Tenorth que nos coloca diante da complexidade das investigações no campo da educação, que nos aponta de um lado a multiplicidade de abordagens e de outro a especificidade associada a esse campo:

Estudos que revelam a diversidade disciplinar e a necessária interdisciplinaridade quando se trata do campo da educação nos fazem levantar a questão da multiplicidade de abordagens, mas também de qual é a especificidade associada ao campo e de sua circunscrição no domínio das ciências humanas e sociais. Há sempre um questionamento, especialmente de cientistas sociais e profissionais da filoso $\neg$ fia, sobre o estatuto da educação como campo de conhecimento. Pergunta-se se há um domínio específico desse campo ou um domínio que assegure de alguma forma sua unidade (fatos, teorias, postulados, etc.) permitindo-lhe, face a outros campos de estudo, conservar uma certa especificidade, tendo a possibilidade de integrar a multiplicidade das disciplinas anexas que o próprio campo cria. (TENORTH, 1998 apud GATTI, 2010, p. 60).

Sem assumir uma unidade de campo como homogeneidade, Gatti (2010) busca uma caracterização mais clara enquanto campo acadêmico e investigativo, tendo uma unidade de propósitos com clareza de abrangência e de estruturas conceituais, mesmo mantendo seu referencial plural. É nesse contexto que trazemos a discussão sobre as formas investigativas e suas questões metodológicas.

Em meio a essa multiplicidade de abordagens metodológicas, quais seriam as formas investigativas reconhecidas nesse campo (ou área) acadêmico e investigativo da educação e como essas formas se organizariam e se comportariam nesse campo? Essas formas garantiriam uma unidade de propósitos e se traduziriam em certa identidade de campo acadêmico e investigativo, conforme posto por essa autora? Ou, contrariamente a essa perspectiva, essas formas investigativas apontariam um novo cenário, o da multirreferencialidade, para a compreensão dos fenômenos educacionais 
(MARTINS, 2004), no qual a busca de uma identidade seria empobrecedora, já que se perderia toda a riqueza e a totalidade desse campo, tão múltiplo, multifacetado e afeito às demandas sociais, que é o da educação?

Ao se pensar na educação ambiental, o exercício de configuração de um campo educacional se amplia, na medida em que há aí um destaque à questão ambiental, conduzindo a discussões sobre: se a EA é parte do campo educacional, se a EA é parte do campo ambiental ou se a EA é parte de ambos, uma intersecção desses dois grandes campos do conhecimento. Compartilhando com Carvalho (2008) a ideia de que a EA é uma área de confluência entre o campo educacional e o campo ambiental e que, por isso, a EA é parte desses dois grandes campos interdisciplinares (KAWASAKI; CARVALHO, 2009), é que situamos as nossas discussões sobre as questões metodológicas nas pesquisas em EA.

Considerando a definição geral de "metodologia de pesquisa" como o processo que descreve a forma como a pesquisa é conduzida, explicitando detalhada e minuciosamente toda a ação desenvolvida no método (caminho) do trabalho de pesquisa quanto ao seu tipo, aos instrumentos utilizados, ao tempo previsto, aos pesquisadores, ao público-alvo, ao tratamento dado aos dados e aos fundamentos e pressupostos filosóficos e teóricos, realizamos o recorte que foi dado às discussões desse GDP: um enfoque inicial às opções metodológicas dos trabalhos apresentados no VI EPEA em relação ao tipo, instrumentos, sujeitos e/ou contextos e temas de pesquisa.

\section{QUE ENCAMINHAMENTOS FORAM DADOS ÀS DISCUSSÕES NO GDP?}

Conforme mencionado, analisamos inicialmente, ou seja, antes da realização do GDP propriamente dito, os cinco trabalhos que foram inscritos nesse GDP, a fim de verificar "quais são as abordagens dadas às questões metodológicas nessas pesquisas" e "em que extensão esses trabalhos apresentam como questão central as questões metodológicas", já que havia uma hipótese de que os trabalhos inscritos nesse GDP apresentariam essa perspectiva de pesquisa. A partir dessa análise, fizemos o planejamento do GDP em questão.

Verificamos que dos cinco trabalhos inscritos nesse GDP (Anexo) quatro apresentavam como questão central aspectos relacionados a questões metodológicas: o trabalho (4), que, ao realizar um estudo do estado da arte da pesquisa em EA analisando teses e dissertações dos Programas de PósGraduação em Educação e Ensino de Ciências da região Centro-Oeste do Brasil, no quinquênio 2006-2010, identifica diferentes tipos de pesquisa (natureza das pesquisas) e diferentes formas e instrumentos de pesquisa; o trabalho (5), que propõe uma metodologia de sensibilização ambiental e um instrumento de pesquisa, um dispositivo audiovisual para a coleta e a produção de dados em EA; 
o trabalho (3), que propõe o uso de metodologias que tragam as subjetividades para o planejamento de ações educativas na perspectiva da EA crítica; e o trabalho (2), que propõe uma abordagem teórico-metodológica freireana, utilizando a metodologia da abordagem temática para o desenvolvimento da pesquisa-ação. Apenas um trabalho (1) não tem como foco as questões metodológicas, propondo-se a tratar dos fundamentos teóricos da Ética, mais especificamente da Bioética, rumo à construção de uma ética ambiental libertadora. Como se pode ver, tais resultados confirmam a hipótese inicial de que os trabalhos inscritos nesse GDP focariam as questões metodológicas das pesquisas em EA. Os títulos e autores desses trabalhos inscritos no GDP estão em anexo a este artigo.

Todavia, considerando que esse GDP é novo e o número pequeno de trabalhos inscritos nele e desconhecendo quais seriam as expectativas dos participantes em relação às discussões que seriam travadas, optamos por estabelecer uma dinâmica que possibilitasse aos participantes exercitar um olhar para o conjunto de 88 trabalhos aprovados no VI EPEA do ponto de vista das opções metodológicas contidas nesses trabalhos.

Dessa forma, nossa proposta se caracterizou pela seguinte dinâmica:

- Preparação coletiva (participantes do GDP) da ficha de análise para os títulos e resumos dos trabalhos apresentados no evento;

- Análise coletiva dos resumos, consultando os trabalhos completos quando necessário. Essa análise foi feita pelos participantes do GDP, divididos em X grupos, e cada grupo analisou Y trabalhos;

- Sistematização das análises pelos coordenadores e discussão coletiva dos resultados.

Os trabalhos foram, então, distribuídos entre os grupos de participantes do GDP, que fizeram a classificação dos resumos de acordo com as seguintes categorias:

1. Natureza da pesquisa: indica o tipo de abordagem metodológica explicitada pelos autores (Ensaio; Estudo de Caso; Pesquisa Etnográfica; Pesquisa-Ação Participante; Pesquisa Fenomenológica; Pesquisa Documental);

2. Instrumentos de pesquisa: indica os instrumentos de pesquisa utilizados na coleta de dados (Entrevista; Pesquisa Documental; Observação Participante; Questionário).

3. Temas de pesquisa: indica os temas ambientais relacionados à atividade de EA investigada no trabalho. Pela dificuldade em desvincular as questões metodológicas das temáticas, estas foram incluídas nessa classificação. 
Os resultados obtidos para cada uma das categorias foram organizados em gráficos para que pudessem ser apreciados pelo grupo. É importante observar que essa classificação foi feita em curto tempo, com base em parâmetros bastante frouxos, sem uma discussão extensa que visasse a padronização dos critérios utilizados pelos participantes do GDP. Assim, os dados que apresentaremos a seguir se constituem em mera aproximação inicial ao perfil metodológico das produções apresentadas no evento, produzida com o único intuito de gerar discussão sobre questões ligadas aos aspectos metodológicos da pesquisa em Educação Ambiental reunida no VI EPEA.

Como primeiro resultado do exercício, como seria esperado, percebemos que havia, entre os pesquisadores reunidos, falta de consenso entre as concepções construídas sobre as diferentes abordagens metodológicas presentes na literatura e observadas no corpo de trabalhos que estava sendo classificado. Ainda assim, foi possível trazer tais diferenças para a discussão e refinar um pouco os resultados obtidos, mas fica clara a necessidade de se ampliar e sistematizar a discussão dos aspectos metodológicos envolvidos na produção de conhecimento no campo da EA.

Outra dificuldade encontrada foi a sobreposição natural que ocorre entre as categorias escolhidas e também entre o que é declarado nos trabalhos em relação à natureza da pesquisa. Tendo em conta que não são categorias mutuamente excludentes, uma vez que se comparam características de diferentes níveis, podese pensar que um trabalho apresentado pelo autor como "estudo etnográfico" poderia também ser classificado como "estudo de caso" (do tipo etnográfico), se esse fosse o caso.

Talvez o grande achado em meio a esse panorama traçado pelos participantes do GDP tenha sido a forte presença de estudos em que a natureza das fontes foi de caráter documental. Severino (2007, p. 122) aponta que na pesquisa documental têm-se como fonte "documentos no sentido amplo, ou seja, não só documentos impressos, mas sobretudo outros tipos de documentos, tais como jornais, fotos, filmes, gravações, documentos legais". Em relação à natureza das pesquisas classificadas, é possível notar certo destaque para estudos que se baseiam em análises documentais:

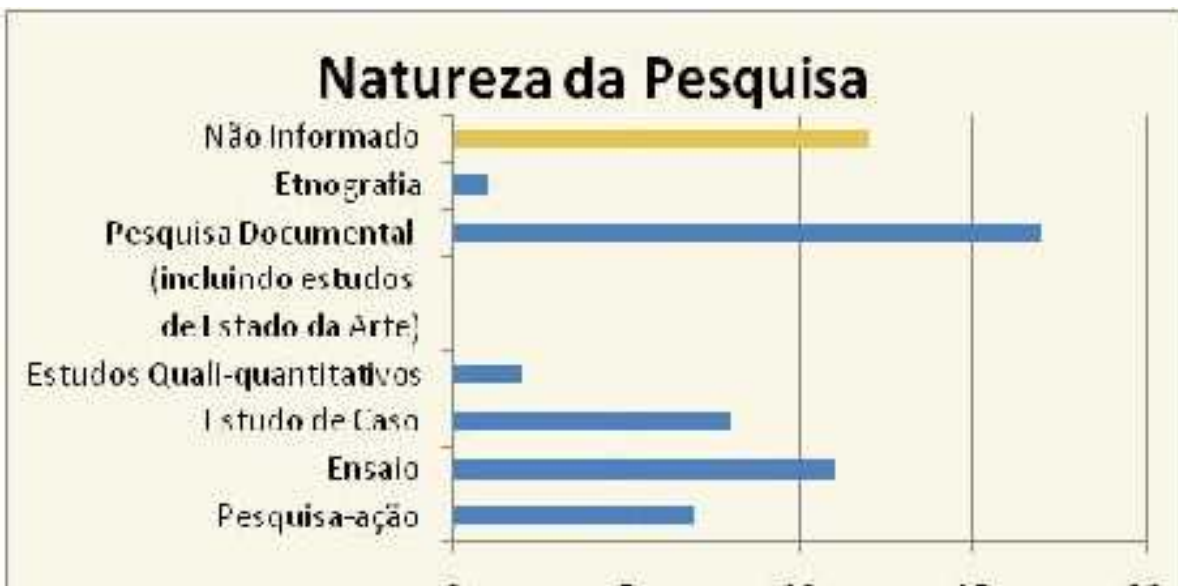


Figura 1 . Distribuição dos trabalhos em relação à natureza da pesquisa.

A forte presença de trabalhos que continham análises documentais fica mais clara quando os participantes apontaram que, em relação aos instrumentos de coleta utilizados, havia grande número de referências a registros documentais:

\section{Instrumentos de Pesquisa}

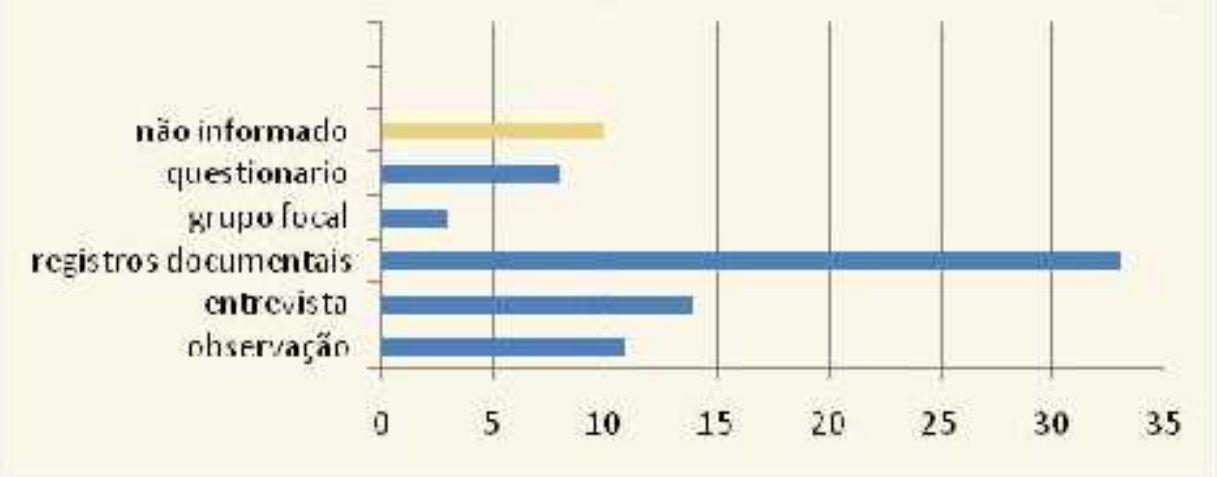

Figura 2. Distribuição dos trabalhos em relação aos instrumentos de pesquisa.

Sem dúvida, a presença de um número expressivo de estudos que se debruçam sobre registros documentais é uma novidade em termos de EPEA e da produção em Educação Ambiental como um todo. A presença de pesquisa documental nos EPEAs já é observada desde a primeira edição, em 2001, como aponta estudo de Freitas e Oliveira (2006), mas se observa, de forma geral, que 
ao longo dos anos as tendências metodológicas dominantes no campo são a pesquisa-ação e a pesquisa participante (AVANZI; CARVALHO; FERRARO JÚNIOR, 2009; PATO; SÁ; CATALÃO, 2009).

No entanto, a natureza das fontes investigadas não nos dá a medida das temáticas contempladas nos trabalhos para que se possa refletir sobre os possíveis significados dessa aparente nova tendência. As temáticas relacionadas aos trabalhos também foram observadas pelos participantes do GDP, o que corrobora a ideia de que pode haver um deslocamento em relação às principais temáticas que vêm sendo abordadas na produção acadêmica em Educação Ambiental.

Podemos perceber, a partir da leitura da Figura 3, que os estudos de "concepções", que tradicionalmente apresentavam elevada frequência nos eventos, aparentemente surgiram em menor número no VI EPEA. Da mesma forma, chama a atenção a presença de 6 trabalhos do tipo "estado da arte", investigando a produção bibliográfica em eventos, periódicos e bancos de teses e dissertações, tratando-se, aparentemente, de uma temática emergente no evento.

\section{Temas de Pesquisa}

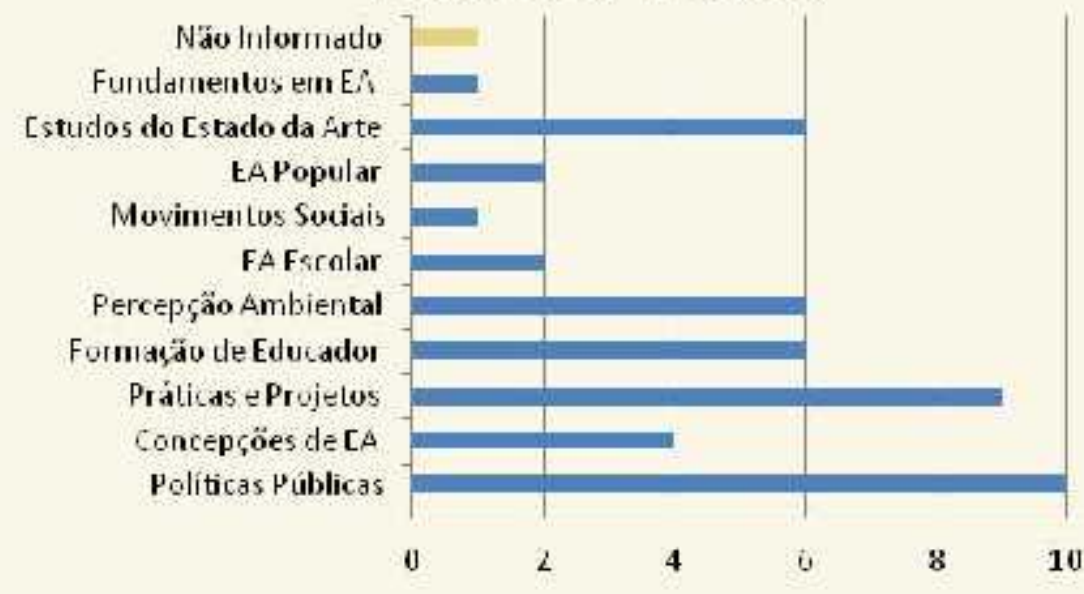

Figura 3. Distribuição dos trabalhos em relação ao tema da pesquisa.

Outra indicação importante no levantamento que fizemos é a forte presença do tema "políticas públicas", que se mostrou o mais frequente em nosso 
levantamento, o que corrobora a ideia de um possível deslocamento das temáticas investigadas no campo da Educação Ambiental ou, ao menos, indica que esse é um tema que vem assumindo maior centralidade e dividindo espaço com temas que ainda continuam muito presentes, como os estudos sobre "percepção ambiental", "formação do educador" e "práticas e projetos em EA".

Percebemos ainda que a somatória de três das temáticas que costumam ou podem pedir, em termos de abordagem metodológica, estudos que envolvam análises documentais - a saber: "estados da arte", "práticas e projetos" e "políticas públicas" - é superior à somatória das demais temáticas. Isso nos leva a suspeitar que o número expressivo de trabalhos que se valeram de registros documentais como fonte de dados esteja relacionado com a frequência dessas temáticas.

Com efeito, em uma leitura dos resumos dos 17 trabalhos indicados pelos participantes do GDP como "pesquisas documentais" em relação à natureza da pesquisa (Figura 1), encontramos 7 trabalhos que abordam políticas públicas, 6 estudos de estado da arte e 4 trabalhos que estudam livro didático ou produto de mídia, o que indica que o aumento da frequência de pesquisas documentais parece estar relacionado com uma maior frequência de trabalhos que abordam tais temáticas.

\section{CONSIDERAÇÕES FINAIS}

A partir desses indícios, fica clara a pertinência de novos estudos que aprofundem, de forma sistematizada, a investigação sobre as tendências atuais da pesquisa em educação ambiental, no âmbito do EPEA e de outros espaços de divulgação da produção acadêmica, procurando-se situar e acompanhar as tendências aqui sugeridas.

O crescimento do número de pesquisas documentais e o possível deslocamento do foco das pesquisas para as políticas públicas e para os estudos de estado da arte podem indicar um momento de maturidade tanto no âmbito da produção acadêmica, que passa a dispor de espaços mais ou menos consolidados de divulgação que são alvo dessas investigações, como no âmbito das políticas públicas específicas de educação ambiental, em que o distanciamento de seus contextos de produção, conferido pelo tempo decorrido desde a implementação dessas políticas, pode favorecer um movimento analítico que antes não seria possível.

Os Grupos de Discussão de Pesquisa que tratam de questões atuais, ditos "GDPs emergentes", sempre passam por um momento de avaliação em termos de sua continuidade na estrutura do EPEA. Em relação ao futuro do GDP "Questões Metodológicas da Pesquisa em Educação Ambiental", acreditamos na pertinência de sua manutenção desde que sejam feitos alguns ajustes e que a 
comunidade o reconheça como um espaço para discutir, de forma mais ampla, as questões decorrentes da multiplicidade de abordagens metodológicas, como já colocamos neste relato.

O reconhecimento das formas investigativas presentes nos trabalhos cujos autores se inscrevem no GDP, além das discussões sobre a identidade de campo ou sobre o cenário da multirreferencialidade, justificaria sua continuidade. Para acompanhar tendências atuais na pesquisa, poderia ser interessante que os participantes também dedicassem um tempo para a apreciação dos resultados apresentados pelos trabalhos de estado da arte presentes no evento. No entanto, para que se possa avançar na profundidade das discussões, será fundamental que o número de trabalhos inscritos nesse GDP se amplie, permitindo um cenário mais representativo, e que os autores efetivamente participem das discussões, sem o que não será possível uma discussão informada e aprofundada.

\section{Referências}

AVANZI, Maria Rita; CARVALHO, Isabel Cristina de Moura; FERRARO JÚNIOR, Luiz Antonio. Um olhar para a produção de pesquisa em educação ambiental a partir do GT Ambiente, Sociedade e Educação, da ANPPAS. Pesquisa em Educação Ambiental, v. 4, n. 2, p. 79-93, 2009.

CARVALHO, Isabel Cristina de Moura. Educação Ambiental: a formação do sujeito ecológico. 4. ed. São Paulo: Cortez, 2008.

CARVALHO, Luiz Marcelo de et al. Relatório Científico do I Encontro de Pesquisa em Educação Ambiental, 2001.

CARVAlHO, Luiz Marcelo et al. Relatório Científico do IV Encontro de Pesquisa em Educação Ambiental, 2007.

FREITAS, Denise de et al. Relatório Científico do II Encontro de Pesquisa em Educação Ambiental, 2003.

FREITAS, Denise de; OLIVEIRA, Haydée Torres de. Pesquisa em Educação Ambiental: um panorama de suas tendências metodológicas. Pesquisa em Educação Ambiental [online], v. 1, n. 1, p. 175-191, 2006.

GATTI, Bernadete Angelina. Reflexões sobre questões metodológicas e práticas em pesquisas em educação. Filosofia e Educação [online], v. 2, n. 2, p. 53-73, out. 2010. 
KAWASAKI, Clarice Sumi et al. Relatório Científico do III Encontro de Pesquisa em Educação Ambiental, 2005.

KAWASAKI, Clarice Sumi; CARVALHO, Luiz Marcelo de. Tendências da Pesquisa em Educação Ambiental. Educação em Revista, v. 25, n. 3, p. 143-157, dez. 2009.

MARTINS, João Batista. Contribuições epistemológicas da abordagem multirreferencial para a compreensão dos fenômenos educacionais. Revista Brasileira de Educação. n. 26, p. 85-94, maio/ago. 2004.

MATOS, Mauricio et al. Relatório Científico do VI Encontro de Pesquisa em Educação Ambiental, 2011.

OLIVEIRA, Haydée Torres de et al. Relatório Científico do V Encontro de Pesquisa em Educação Ambiental, 2009.

PATO, Claudia; SÁ, Lais Mourão; CATALÃO, Vera Lessa. Mapeamento de tendências na produção acadêmica sobre educação ambiental. Educação em Revista, v. 25, n. 2, p. 213-233, dez. 2009.

SEVERINO, Antonio Joaquim. Metodologia do trabalho científico. São Paulo: Cortez, 2007. 304 p.

\section{ANEXO: TRABALHOS INSCRITOS NO GDP}

1) BIOÉTICA: APORTES PARA UMA ÉTICA AMBIENTAL LIBERTADORA (COSTA, César Augusto Soares).

2) A CONCEPÇÃO EDUCACIONAL FREIREANA E O CONTEXTO ESCOLAR: SUBSÍDIOS À EFETIVAÇÃO DAS DIMENSÕES "PESQUISA E AÇÃO” EM EDUCAÇÃO AMBIENTAL NA ESCOLA (TORRES, Juliana Rezende; MAESTRELLI, Sylvia Regina Pedrosa).

3) DIÁLOGO SOBRE PERCEPÇÃO E METODOLOGIAS NA EDUCAÇÃO AMBIENTAL (GUIMARÃES, Mauro; SÁNCHEZ, Celso).

4) PESQUISA EM EDUCAÇÃO AMBIENTAL NO CENTRO-OESTE BRASILEIRO: UM ESTUDO DA PRODUÇÃO ACADÊMICA DAS ÁREAS DE EDUCAÇÃO E ENSINO DE CIÊNCIAS (BARBOSA, Leila Cristina Aoyam; ZANON, Angela Maria).

5) A PRODUÇÃO DE DADOS E A PESQUISA EM EDUCAÇÃO AMBIENTAL ATRAVÉS DE UM DISPOSITIVO AUDIOVISUAL (AZEVEDO, Cláudio Tarouco de; MARTIN, Alfredo Guillermo).

Artigo recebido em 26/10/2012

Artigo aprovado em 03/12/2012 